Communication

\title{
SWNTs-catalyzed solar hydrogen production
}

\section{Young Kwang Kim ${ }^{\S}$, Gulzar Khan ${ }^{\S}$, Hye Won Jeong ${ }^{\S \S}$, and Hyunwoong Park ${ }^{\S \S *}$}

\author{
${ }^{\S}$ Department of Physics, ${ }^{\S}$ School of Energy Engineering, Kyungpook National University, Deagu 702-701, Korea
}

\begin{abstract}
Single-walled carbon nanotubes (SWNTs) catalyzed hydrogen production from water containing various electron donors under visible light $(\lambda>420 \mathrm{~nm})$. As-received SWNTs were effective for hydrogen production, yet the effect vanished when they underwent surface chemical treatments. Upon coupling with CdSe particles, however, the surface treated SWNTs were far superior to non-treated SWNTs by a factor of $\sim 30$ for hydrogen production.
\end{abstract}

Solar hydrogen production has been received wide attention over the past four decades particularly in semiconductor systems. ${ }^{1}$ Unfortunately, the benchmark solarto-hydrogen (STH) efficiency is still as low as $\sim 2 \%$ even though complicated materials and systems are designed. For high STH efficiency, semiconductors of interest should absorb wide spectrum of sunlight, display efficient charge separation and transfer, and effectively catalyze water splitting. ${ }^{2}$ In the latter, many auxiliary metals and metal oxides $^{3-5}$ have been coupled to base semiconductors to hurdle the uphill activation energy barrier. Recently, inexpensive carbon materials including nanotubes, nanofibers, graphites, and graphenes have been found to effectively catalyze hydrogen production from water. ${ }^{6-10}$ The electric conductivity of carbon materials was found to be a primary factor in determining the STH efficiency of semiconductor/carbon composites. ${ }^{10}$ Among these carbon materials, single-walled carbon nanotubes (SWNTs) were found to be capable of producing hydrogen under visible light even in the absence of semiconductor particles. The hydrogen production was significantly affected by electron donors, while coupling of Pt particles on SWNTs reduced $\mathrm{H}_{2}$ production.

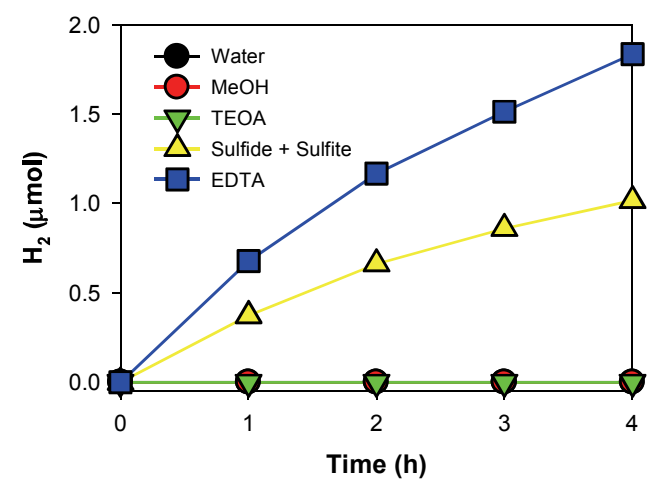

Figure 1. Time-profiled hydrogen productions in aqueous SWNT suspensions with electron donors (ED) under visible light $(\lambda>420 \mathrm{~nm}) .[\mathrm{SWNT}]=1 \mathrm{~g} \cdot \mathrm{L}^{-1} ;[\mathrm{ED}]=0.1 \mathrm{M} ; \mathrm{N}_{2^{-}}$ purged for $30 \mathrm{~min}$ prior to photocatalysis. $\mathrm{MeOH}$, TEOA,

*To whom correspondence should be addressed. E-mail: hwp@knu.ac.kr and EDTA refer to methanol, triethanolamine, and ethylenediaminetetraacetate, respectively, while water indicates no addition of ED.

SWNT powders (inner diameter: $1 \sim 1.2 \mathrm{~nm}$, length $\sim 20$ $\mu \mathrm{m})$ (ASA-100F, Hanwha Nanotech) were used as received or after treatments with acid and heat. For the acid treatment, SWNTs $\left(1 \mathrm{~g} \cdot \mathrm{L}^{-1}\right)$ were suspended and stirred in an aqueous solution of $\mathrm{HCl}$ and $\mathrm{HNO}_{3}$ at a volumetric ratio of $3: 1$ for 30 min. Then the samples were rinsed with distilled water several times, filtered with $0.45-\mu \mathrm{m}$ nitrocellulose membrane filters, and dried under air. For the heat treatment, SWNT powder was annealed at $300{ }^{\circ} \mathrm{C}$ for $10 \mathrm{~min}$ in the presence of air. SWNTs were also loaded with platinum nanoparticles via a chemical reduction method. Platinum precursor $\left(\mathrm{H}_{2} \mathrm{PtCl}_{6}\right)$ was dissolved at $0.5 \mathrm{wt} \%$ with respect to SWNT in aqueous SWNT suspensions $\left(0.5 \mathrm{~g} \cdot \mathrm{L}^{-1}\right)$ and stirred for 3 hours. Then, borohydride salt $\left(\mathrm{NaBH}_{4}, 2 \mathrm{~g} \cdot \mathrm{L}^{-1}\right)$ as a reducing agent was added to the suspension under vigorous stirring, resulting in the reduction of platinum on the SWNT framework. SWNTs were also coupled to $\mathrm{CdSe}$ particles by following a method found elsewhere. ${ }^{10}$ Photocatalytic reactions were completed by suspending bare and modified SWNT particles (SWNT, aSWNT, h-SWNT, SWNT/Pt, and SWNT/CdSe) in water containing various electron donors (methanol, triethanolamine, ethylenediaminetetraacetate, and sulfide/sulfite mixture; each $0.1 \mathrm{M}$ ) at $1 \mathrm{~g} \cdot \mathrm{L}^{-1}$. Nitrogen gas purged through the suspensions for $30 \mathrm{~min}$, and then visible light $(\lambda>420 \mathrm{~nm})$ was irradiated to the glass reactor equipped with a quartz disc for light penetration by inserting a long-wave pass filter between the reactor and a solar simulator with AM 1.5G filter (LS-150 Xe, Abet Technology). The headspace gas was intermittently sampled and analyzed for molecular hydrogen using a gas chromatograph device (ACME 6100, Youngling Instrument) equipped with a thermal conductivity detector.

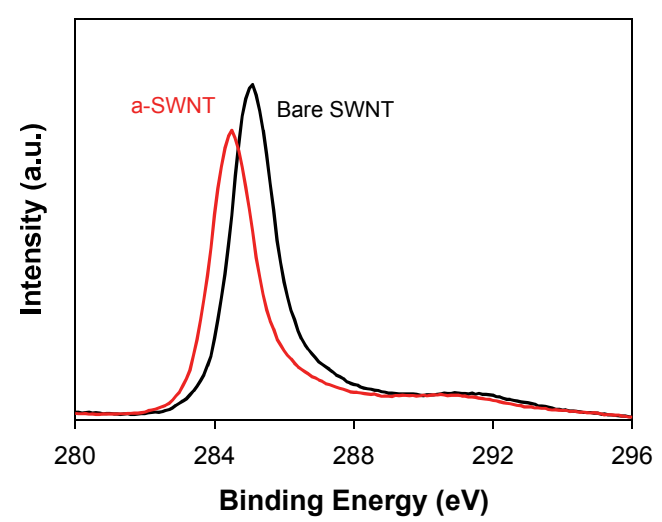

Figure 2. XPS spectra of $\mathrm{C} 1 \mathrm{~s}$ for bare and acid-treated SWNTs. 
Figure 1 shows the effects of electron donors on the hydrogen production in SWNT suspensions under visible light. In the absence of any electron donor (i.e., pure water), no hydrogen was produced due to the small bandgap of SWNTs $(<1 \mathrm{eV})$. The addition of electron donors with redox potentials negative of $\mathrm{E}^{\circ}\left(\mathrm{O}_{2} / \mathrm{H}_{2} \mathrm{O}\right)$ can drive hydrogen production from water although the LUMO level of SWNTs is comparable to $\mathrm{E}^{\circ}\left(\mathrm{H}_{2} \mathrm{O} / \mathrm{H}_{2}\right)$. Methanol $(\mathrm{MeOH})$ and triethanolamine (TEOA) were found to be ineffective, whereas $\mathrm{H}_{2}$ production was significantly enhanced in the presence of sulfide/sulfite mixture and EDTA. The comparison of electrochemical redox potentials indicated that EDTA is the most difficult to be oxidized followed by TEOA, sulfide, and methanol.

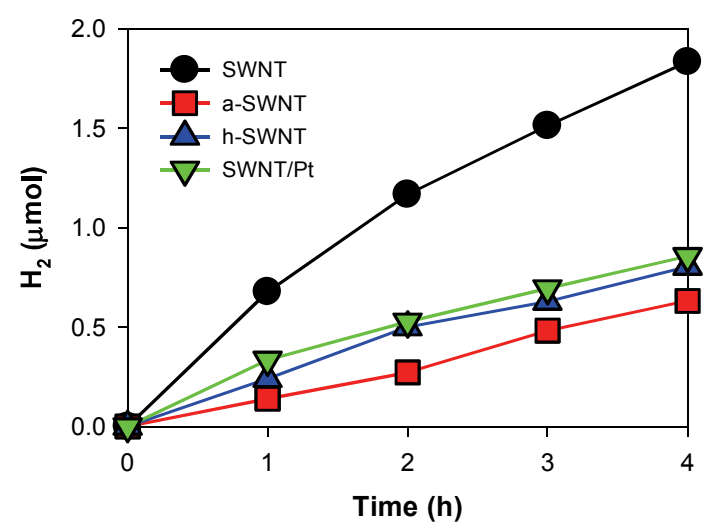

Figure 3. Effects of surface treatment on the hydrogen production in SWNT suspensions $\left(1 \mathrm{~g} \cdot \mathrm{L}^{-1}\right)$ with EDTA $(0.1$ M) under visible light $(\lambda>420 \mathrm{~nm}) . \mathrm{N}_{2}$-purged for $30 \mathrm{~min}$ prior to photocatalysis. a-SWNT and h-SWNT refer to acidtreated and heat-treated SWNTs, respectively.

Nevertheless, the highest effect of EDTA for inducing $\mathrm{H}_{2}$ evolution was attributed to its strong interaction with SWNTs. XPS study showed that bare and acid-treated SWNTs have diverse functional groups including ether, ketone, quinine, carboxyl, carboxylic anhydride, and esters (Figure 2). Meanwhile, the C1s spectrum for a-SWNTs was shifted to low energy by $\sim 0.6 \mathrm{eV}$, suggesting that a-SWNTs have pronounced $\mathrm{sp}^{2}$ carbons and/or the acid treatment makes SWNTs reduced. In either case, bare SWNTs are likely to have stronger interaction with positively charged EDTA, forming new bonds capable of being locally photoexcited. On the other hand, the other electron donors appeared to have less interaction with SWNTs. The higher effect of sulfide/sulfite mixture than those of methanol and TEOA was associated likely to rapid regeneration of oxidized sulfide (e.g., polysulfides) by sulfite.

Figure 3 compares the effect of surface-treated SWNTs for hydrogen production. As compared to bare SWNTs, acid treatment of SWNTs reduced the $\mathrm{H}_{2}$ production while heat treatment was found to be ineffective as well. The reduced activities of acid and heat-treated SWNTs were attributed to decreased interaction with EDTA, while the latter would cause some damage of SWNT frame as well. Surprising is that even $\mathrm{Pt}$ loading decreased the $\mathrm{H}_{2}$ production performance of bare SWNTs. This strongly indicates that the SWNTs surface is very delicate to chemical treatments, significantly influencing the photo-activity of SWNT. It has been shown that surface oxidation of CNTs by annealing and acid treatment remove disordered or dangling carbons. ${ }^{6-10}$ These treatments increased the graphitic property and electrical conductivity, whereas surface area was not altered significantly. Borohydride treatment (for Pt loading) also could affect the surface carbon states in a similar way. The hydrogen production in SWNT suspension under irradiation, therefore, is not truly photocatalytic and can be associated with a complicated surface carbon photochemistry.

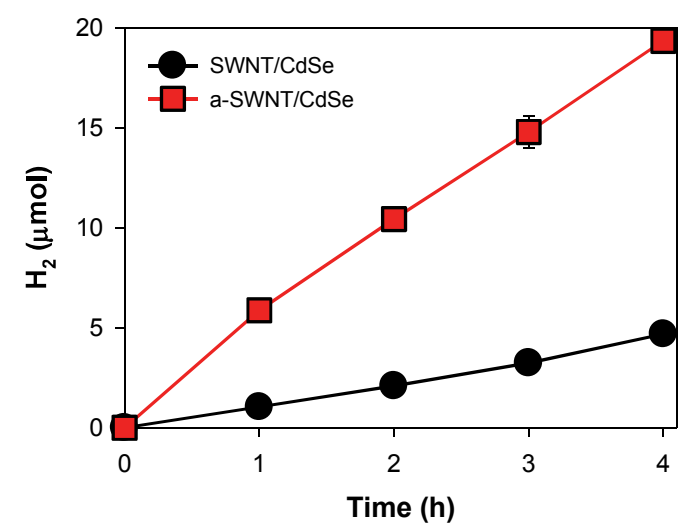

Figure 4. Effects of surface treatment on the hydrogen production in SWNT/CdSe suspensions $\left(1 \mathrm{~g} \cdot \mathrm{L}^{-1}\right)$ with EDTA $(0.1 \mathrm{M})$ under visible light $(\lambda>420 \mathrm{~nm}) . \mathrm{N}_{2}$-purged for 30 min prior to photocatalysis. a-SWNT refers to acid-treated SWNTs.

When SWNTs are coupled to semiconductor particles, the surface treatment effect of SWNTs becomes clear. Figure 4 compares the hydrogen production in bare and acid-treated SWNT-coupled CdSe particles suspended in aqueous EDTA solution under visible light. Upon coupling to CdSe, the hydrogen production with bare SWNTs was enhanced by a factor of $\sim 2$. On the other hand, the acid treatment of SWNTs increased the hydrogen production in CdSe composites by a factor of $\sim 30$ (Figures 3 vs. 4). No significant change in $\mathrm{Cd}^{2+}$ adsorption (i.e., degree of surface CdSe formation) was found. In this composite, the primary role of SWNTs is a hydrogen evolution reaction (HER) catalyst and the catalytic effect becomes greater with surface treatment. Bare SWNTs with surface carbon impurity or dangling carbons can drive proton reduction in the presence of suitable electron donors. These carbons coupled with suitable electron donors can serve as chromophoric centers, whereas surface treatments remove the carbons and make SWNTs less photoactive. Upon coupling with CdSe, the surface carbons of bare SWNTs block a number of photons to be absorbed to CdSe. On the other hand, surface treatment strengthens the graphitic property, increases the electrical conductivity of SWNTs, and effectively removes the impure carbon interference effect. As a result, photogenerated electrons of CdSe are more effectively transferred to protons enhancing hydrogen production.

KEYWORDS: Carbon materials, Photocatalyst, Hydrogen production, Surface treatment, Electrocatalyst.

Received September 11, 2014; Accepted September 17, 2014. 


\section{ACKNOWLEDGEMENT}

The authors thank the National Research Foundation for Global Research Network Program (NRF2014S1A2A2027802) and Space Core Technology Development Program (NRF-2014M1A3A3A02034875), Korea. In addition, we are grateful to the Korea CCS R\&D Center (KCRC) (No. 2014M1 A8A1049354).

\section{REFERENCES AND NOTES}

1. Chen, X. B.; Shen, S. H.; Guo, L. J.; Mao, S. S. Chem. Rev 2010, 110, 6503-6570.

2. Jeong, H. W.; Jeon, T. H.; Jang, J. S.; Choi, W.; Park, H. J. Phys. Chem. C 2013, 117, 9104-9112.

3. Choi, S. K.; Choi, W.; Park, H. Phys. Chem. Chem. Phys. 2013, 15, 6499-6507.
4. Choi, S. K.; Kang, U.; Lee, S.; Ham, D. J.; Ji, S. M.; Park, H. Adv. Energy Mater. 2014, 4, 1301614.

5. Jeon, T. H.; Choi, W.; Park, H. Phys. Chem. Chem. Phys. 2011, 13, 21392-21401.

6. Jeong, H. W.; Park, H. Catal. Today 2014, 230, 15-19.

7. Khan, G.; Choi, S. K.; Kim, S.; Lim, S. K.; Jang, J. S.; Park, H. Appl. Catal. B 2013, 142, 647-653.

8. Khan, G.; Kim, Y. K.; Choi, S. K.; Han, D. S.; AbdelWahab, A.; Park, H. Bull. Korean Chem. Soc. 2013, 34, 1137-1144

9. Kim, Y. K.; Park, H. Energy Environ. Sci. 2011, 4, 685694.

10. Kim, Y. K.; Park, H. Appl. Catal. B 2012, 125, 530-537. 